\title{
Time varying mass and inertia in paper winding multibody simulation
}

\author{
Edo Drenth \\ Modelon AB \\ Ideon Science Park, Lund, Sweden \\ edo.drenth@modelon.com
}

\begin{abstract}
This paper will discuss Modelica's unprecedented flexibility for multi-body simulations. Classical multi-body simulation has as a prerequisite constant mass and inertia for deriving the equations of motion for rigid bodies. However, there are industry applications, like the control development of paper winding, that require time dependency of mass and inertia. In these applications mass and inertia cannot be assumed constant and will thus constitute part of the differential equations system by means of introducing mass and inertia as states.
\end{abstract}

Introducing mass and inertia as states, rather than parameters, requires reformulation of the Newton/Euler formulation of the body model component in the Modelica mechanics multibody library [3].

A successful new body model formulation has been created and is applied in an industrial example system model.

Keywords: dynamic mass, dynamic inertia, multibody, mechanics, paper winding, vibration, FMI

\section{Introduction}

In the paper industry winding machines are used to reduce the inconveniently large paper roll into smaller paper rolls of just a few tons. The dynamic properties of these machines are heavily influenced by the change in mass and inertia of the paper rolls while winding and unwinding $[1,2]$. The time varying resonance frequencies of the system will put limits on the machines throughput.

The paper industry has an interest to investigate the dynamic machine properties by simulation as the references are proof of. This publication will deal with one of the key aspects of a simulation package to handle; the mass and inertia time (revolution) dependency.

Many specialised multi-body packages are built upon constant mass and inertia's to solve the equations of motion. To coop the problem of varying mass, the system is analysed at different points of operation rather than simulating a full run.

Mastering this topic of dynamic mass and inertia properties may not only allow for system controllers' validation in the time domain with Dymola's ${ }^{1}$ real time capabilities, but also support algorithm development with FMI [6] technology exporting models to control development environments.

This publication shows Modelica's capabilities in to this specific topic of paper winding.

\section{Modelica Body}

\section{Mass rate signals}

In order to create sound models, which can be diagnosed upon dimension consistency, mass rate signals are defined. These read,

type MassRate $=$

Real (final quantity="MassRate", final unit= "kg/s");

\footnotetext{
${ }^{1}$ Dymola is a registered trademark of Dassault Systèmes
} 
type MomentOfInertiaRate $=$

Real (final quantity="MomentOfInertiaRate", final unit= " kg.m2/s");

\section{Newton/Euler equations}

The Modelica Standard Library defines bodies with the help of Newton/Euler equations around the centre of mass of the modelled body. These equations have to be elaborated to count for the mass and inertia rates. From Newton's second law we have

$$
\begin{gathered}
p=m v \\
F_{\text {net }}=\frac{d p}{d t}=m \frac{d v}{d t}+v \frac{d m}{d t}
\end{gathered}
$$

In the above equation $F_{\text {net }}$ is the net external force applied to the system, since Newton's second law is only valid for constant mass particles [3]. Reference [4] exemplifies how a net force can be derived, hence

$$
F_{\text {ext }}+m_{w} \frac{d u}{d t}+u \frac{d m}{d t}=m \frac{d v}{d t}+v \frac{d m}{d t}
$$

The net force on the left hand side of the above equation includes a so called "thrust" force from the mass flow, $\frac{d m}{d t}$. In our application a difficulty arises to determine the web mass, $m_{w}$, which is accelerated. At constant web velocity, $u$, this term vanishes.

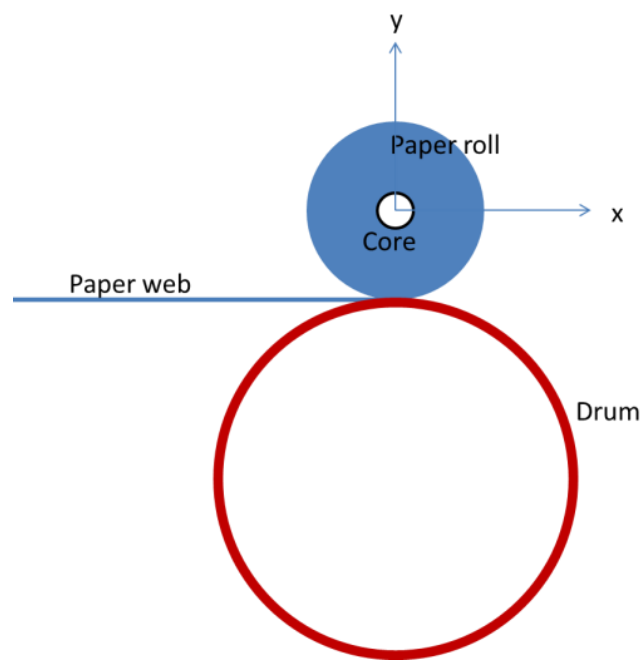

Figure 1 Sketch of model
With help of the right hand side of the force equation, the Modelica code for a body in 3D will be modified and yields,

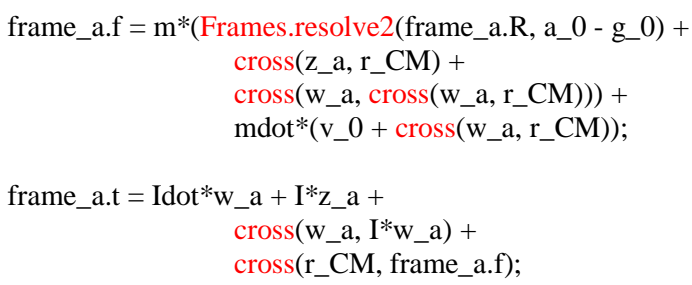

In the above set of equations mdot will defined as mass rate and Idot as a $3 \times 3$ inertia tensor with pivots of moment of inertia rate signals. The above mentioned "thrust" force from reference [4] will have to be applied externally to frame_ $a$ as forces and moments.
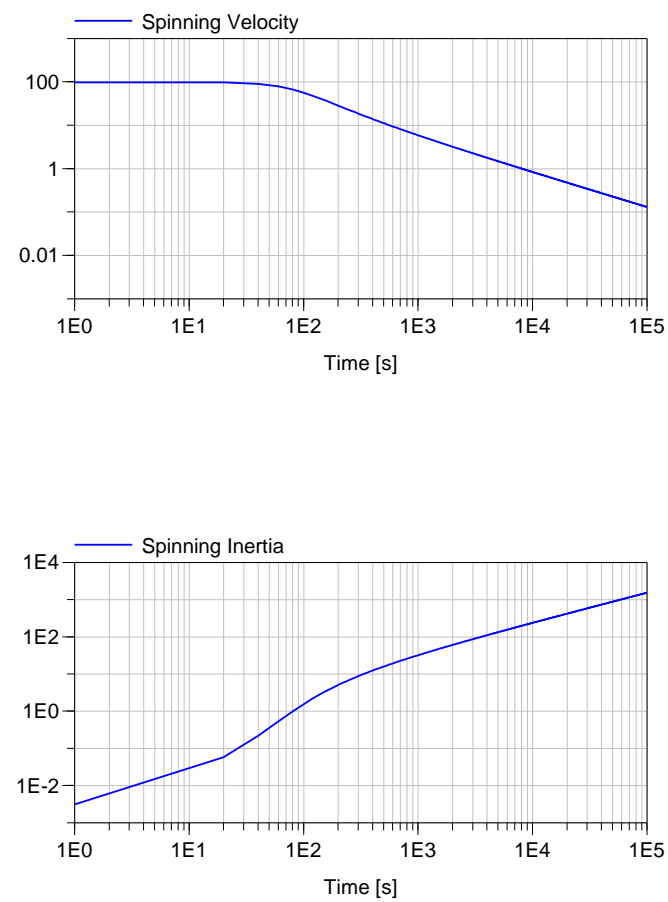

Figure 2 Free spinning results with constant impulse momentum

\section{Body properties}

A special variable mass and inertia model has been designed that can resemble the effects of increasing radius of the paper winding roll. The radius (rate) of this body defines both mass (rate) and moment of inertia (rate) too. The inertia tensor however, and especially the rate, is application dependent and a generic solution difficult. Hence, the paper deals with paper winding only. All properties are dynamically sound for a multi-body analysis. 
E.g. a free winding roll with initial velocity will reduce angular velocity over time due to increased spinning moment of inertia (see Figure 2) at constant momentum (net external force equals zero).

Difficulties arose by straight forward modelling of variable mass and inertias. The symbolic manipulation of the equations showed imbalance on the number of equations and unknowns. These are overcome with simple and physical sound, mathematical reformulation of the existing body model in the Modelica Standard Library.

This dynamic mass and inertia body model is accessible from a library and has become a building block for usage in other models for mechanical simulation.

\section{Model Assumptions}

The model envisioned shall deal with the simulation problem of variable mass and inertia solely. One drum, with the drive input, and one paper roll (blue coloured in the animation series below) are modelled. The interaction between the drum and paper roll is based upon a simple impact force function for the support forces and a simple load dependent slip force with relaxation for the horizontal forces (see Figure 3). These horizontal forces will apply a drive torque on the paper roll and a torque load on the driving drum.

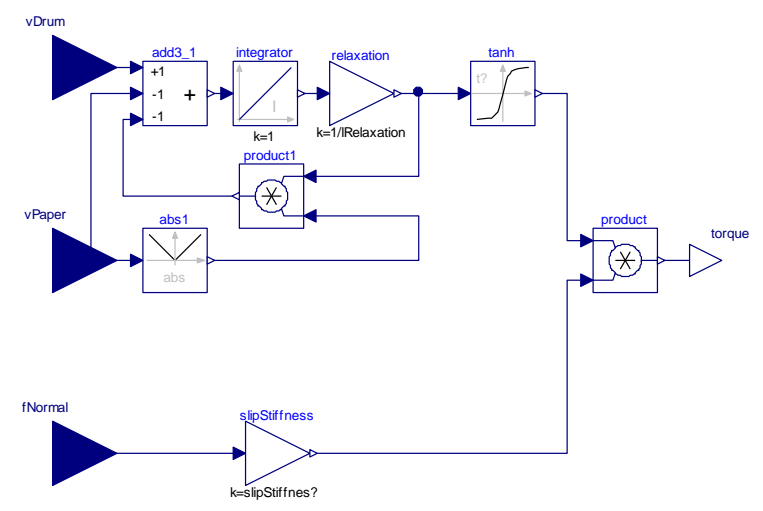

Figure 3 Load dependent slip force with relaxation

No special emphasis is made on material properties of paper with respect to friction and material damping, nor the web tension's influence on the dynamic performance. The models are purely made to show Modelica's capabilities of the specific problem of variable mass and inertia.

The paper roll can move freely in the vertical direction and around its spin axis only, for reasons of simplicity and the limited scope of this report the other four degrees of freedom are kinematic constraint.
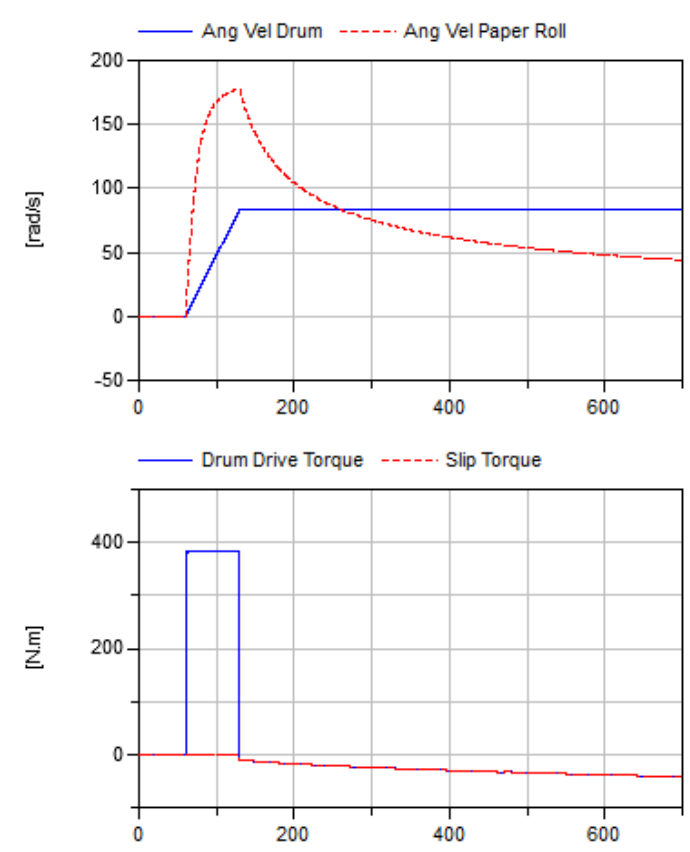

Figure 4 Speed and Torque profile of Drum and Paper Roll

\section{Load case}

The drum is driven with a speed profile as indicated in Figure 4. The speed profile equates to certain constant web acceleration until a predefined velocity is reached. This velocity is kept constant throughout the remaining simulation. For the sake of comparison the direction of operation is taken positive, whereas in the real application the drum and paper roll rotate in opposite directions.

The centre of mass of the paper roll has a small offset from the centre line in order to introduce vibrations in the two drum system. These induced vibrations are solely for the sake of exemplifying the time varying oscillations. 

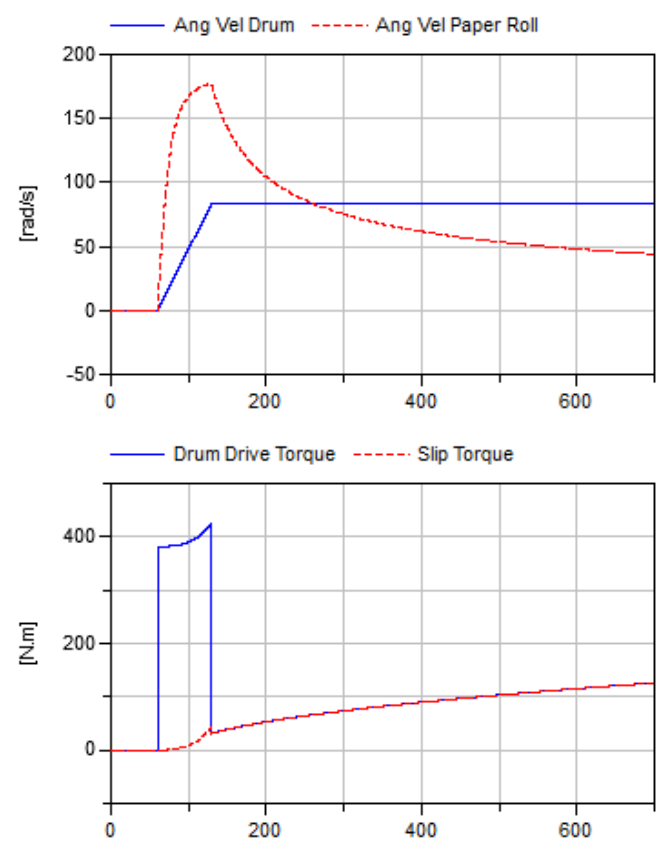

Figure 5 Speed and Torque profile of Drum and Paper Roll

\section{Discussion of Results}

\section{Speed and Load}

The drum is driven with a speed profile as indicated in Figure 4, the paper roll angular velocity is determined through acceleration of the same by means of a frictional force at the interfacing surface of roller and drum. Because the radius of the paper roll starts at the core radius, which is much smaller than the drum's radius, and increases over time the angular velocity increases steeper than the drum's angular velocity. At approximately $260 \mathrm{~s}$ (with the used data) both radii are equal and hence the angular velocity is equal too.

The speed profile of the drum will require a driving torque as indicated in Figure 4 above. The initial large value is due to the acceleration of the heavy drum. Once the desired velocity of the web is reached the torque level is determined by the increase of the paper roll spinning inertia. This paper roll inertia increment becomes a torque load (red in Figure 4) upon the system. Surprisingly the actual load is negative, because the incoming paper from the web results in a system accelerating torque from the linear momentum (web tension omitted), because its forces are applied tangential at the paper roll surface. To keep a constant web velocity the driving drum torque is negative.
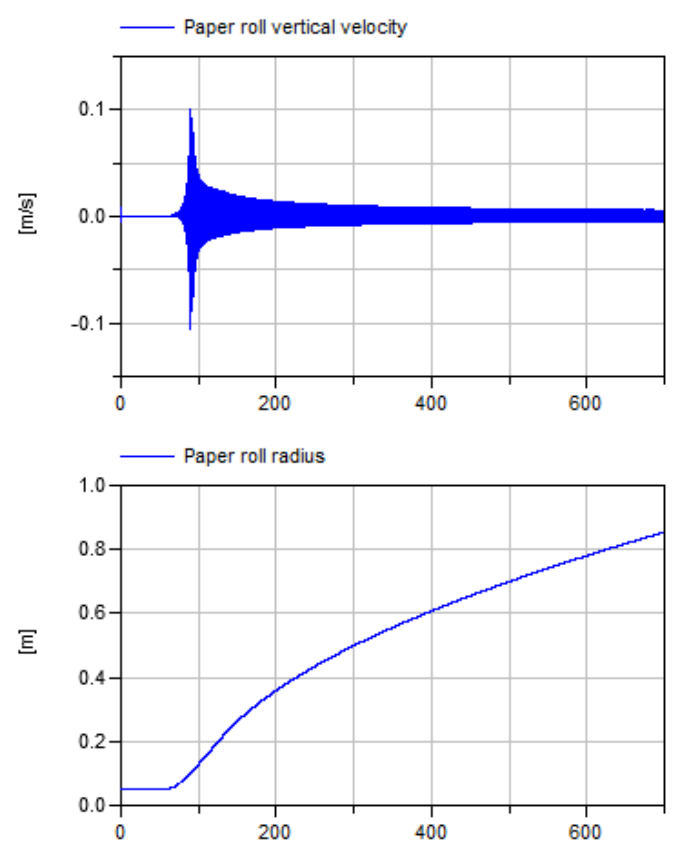

Figure 6 Roller vertical velocity and radius over time

Figure 5 depicts results when the web linear momentum is omitted. The increasing inertia yields an increasing torque.

The offset on the centre of gravity results in a forced vibration of the paper roll in the vertical direction (Figure 6) and around its spin axis (Figure 7). The paper roll is constraint in the remaining directions, but could technically have compliant bearings. This is outside the scope of this paper.

The radius of the paper roll increases over time and the increasing radius will also create vertical motion of the centre of gravity. The mean value of the vertical velocity is positive indicating the centre of gravity rises.

\section{Radius and velocity}

The radius rate of change decreases over time, because the web velocity is constant and the actual radius increases. The paper roll's tangential velocity is constant, hence the angular velocity must decrease and thus the radius rate. 

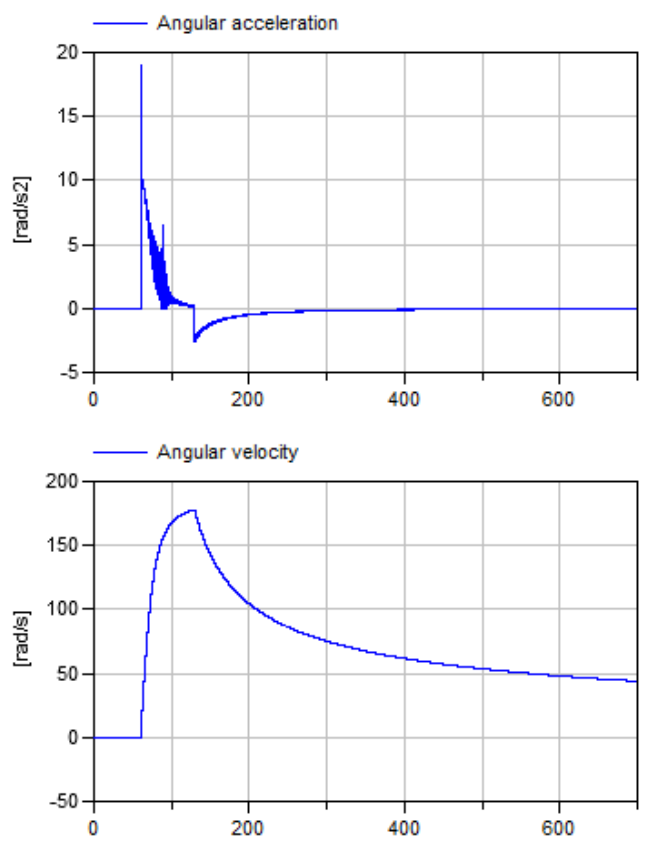

Figure 7 Roller angular velocity and acceleration

\section{Impact Force}

The paper roll is supported with help of an impact force between the drum and the paper roll. Due to the fact the mass increases over time the impact force will increase too (see Figure 8). A close up is made to show the lift of at around $90 \mathrm{~s}$ of simulation time.

Do mind that the impact function, with viscous damping only, may not at all be representative for paper properties. Modelica is however, very well suited to accommodate any impact model and thus able to model material damping, but again outside the scope of this paper.

\section{Bending and torsion}

The discussed and simulated model has neither bending compliance nor torsional compliance. These affects are omitted in this paper, but will have an impact on the dynamic behaviour of the real system. One could however, make a roller and a drum component that can be compliant connected to another roller and drum component easily in Modelica. This way a discretized lumped mass and inertia roller and drum system is created to reflect the bending and torsional vibrations of the system.
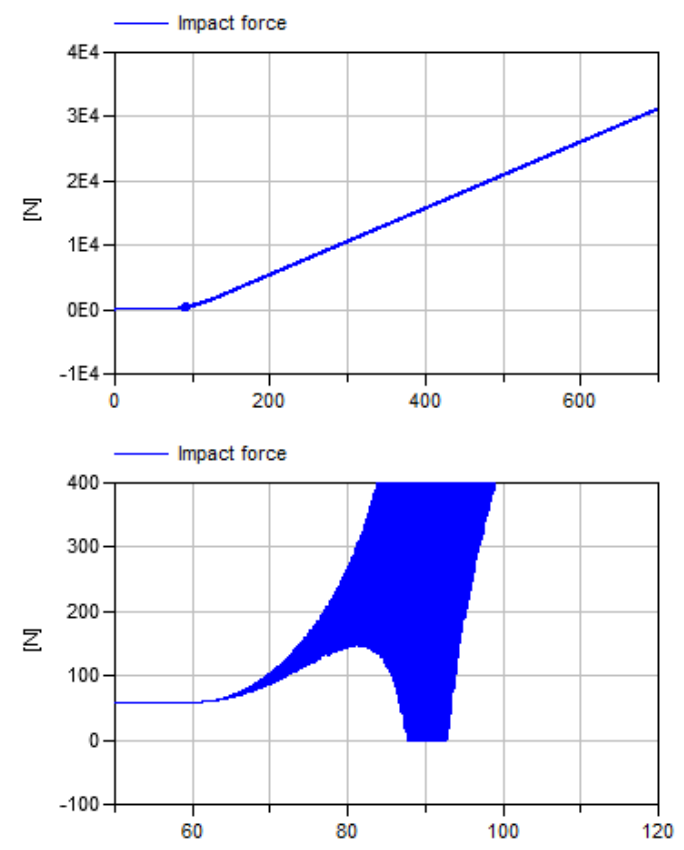

Figure 8 Impact force between drum and paper roll

\section{Animation}

Dymola has capabilities of dynamic graphical presentation of the variable mass and inertia body. Below an animation sequence with a sample every $100 \mathrm{~s}$ is presented of the simulation run discussed in this paper. 


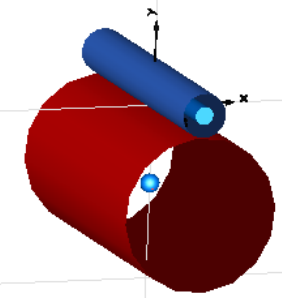

Figure 10 Animation, $t=100 s$

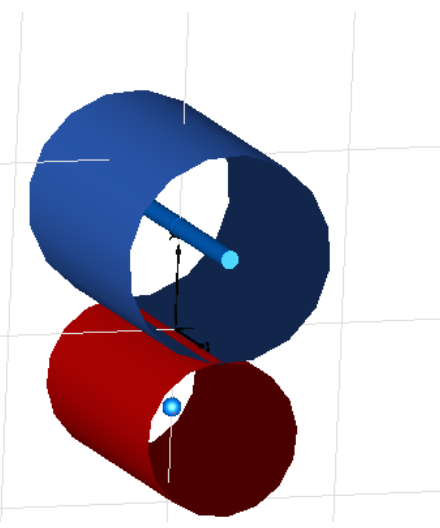

Figure 13 Animation, $t=400 s$

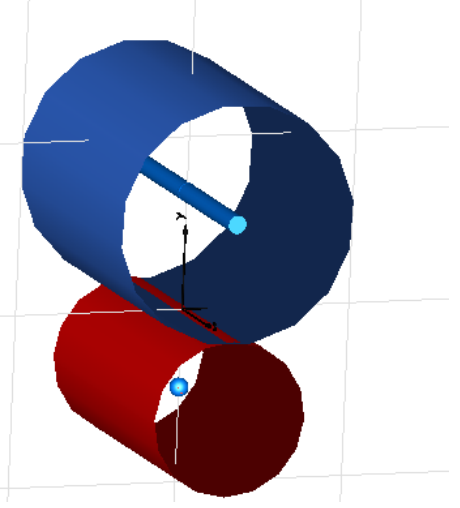

Figure 14 Animation, $t=500 \mathrm{~s}$

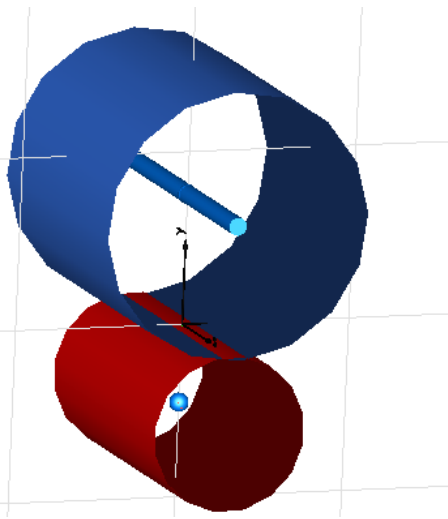

Figure 15 Animation, $t=600 \mathrm{~s}$

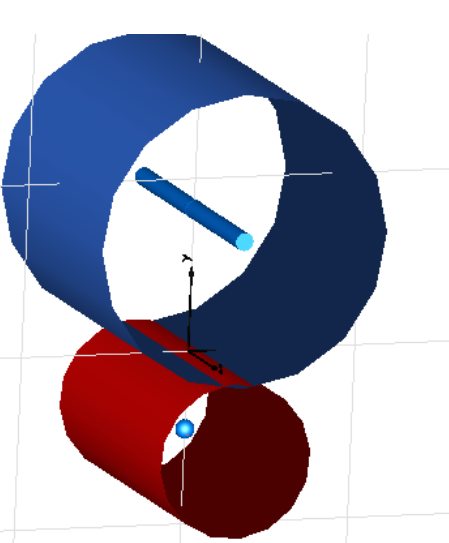


Figure 16 Animation, $\mathrm{t}=700 \mathrm{~s}$

\section{Functional Mock-up Interface}

The newly developed dynamic mass and inertia models can also be used for controller development in specialised environments like Simulink $^{2}$. The FMI Toolbox from Modelon is used to import the Functional Mock-up Unit exported from Dymola. The result is depicted in Figure 17.

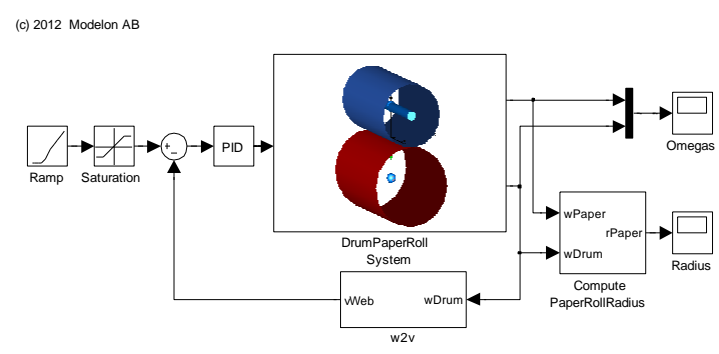

Figure 17 Simulink model with FMI block of the Drum-Paper Roll mechanism

This model exchange allows the control engineer to have an excellent non-linear plant model to develop his algorithms against.

Dymola's real time capabilities allow the user to export the models to a HIL system and verify the actual electronic controller.

\section{Conclusions}

This report shows that Modelica is capable of modelling variable mass and inertias of winding machinery. A special body element is created in the Modelica language which becomes a reusable body object. This body can be used to model a lumped system of a roller to accommodate bending and torsional modes (future work).

The created dynamic mass and inertia model is a prerequisite allowing for virtual controller software development, verification and validation at a systems level for a complete winding cycle.

\section{References}

1. Virtanen T, Fault Diagnostics and Vibration Control of Paper Winders, $\mathrm{Ph} \mathrm{D}$ dissertation Helsinki University of Technology, Espoo, Finland, 2006

2. Zwart, J., Tarnowski, W., Winder Vibration Related to Set Throw-outs, PAPTAC $89^{\text {th }}$ Annual Meeting, Montreal, Canada, 2003

3. Kleppner, D, Kolenkow, R., An Introduction to Mechanics, McGrawHill, pp. 133-134, 1973

4. Chandler, D., Newton's Second Law for Systems with Variable Mass, The Physics Teacher, Vol. 38, 2000

5. Modelica Standard Library, Version 3.2

6. Blochwitz, T., Otter, M., Arnold, M., Bausch, C., Clauß, C., Elmqvist, H., Junghanns, A., Mauss, J., Monteiro M., Neidhold, T., Neumerkel, D., Olsson, H., Peetz, J.-V., Wolf, S., The Functional Mockup Interface for Tool independent Exchange of Simulation Models, $8^{\text {th }}$ Modelica Conference, Dresden, Germany, 2011

7. FMI Toolbox User's Guide 1.3.1, Modelon AB, Lund, Sweden, 2012

${ }^{2}$ Simulink is a registered trademark of The Mathworks 
